\title{
Light Emitting Diodes (LEDs)
}

\author{
K.V.S.Sreedhar \\ ECE Dept. /Gayatri College of Engg/Visakhapatnam, India
}

\begin{abstract}
This paper is to explain the theory and the underlying principle behind the functioning of an LED.
I. Introduction

In the mid 1920s, Russian Oleg Vladimirovich Losev independently created thefirst LED, although his research was ignored at that time.In 1955, Rubin Braunstein of the Radio Corporation of America reported on infrared emission from gallium arsenide (GaAs) and other semiconductor alloys. Experimenters at Texas Instruments, Bob Biard and Gary Pittman, found in 1961that gallium arsenide gave off infrared radiation when electric current was applied. Biard \& Pittman received the patent for the infrared light-emitting diode. In 1962, Nick Holonyak Jr., of the General Electric Company and later with the University of Illinois at UrbanaChampaign, developed the first practical visible spectrum LED. He is seen as the "father of the light-emitting diode". In 1972, M. George Craford, Holonyak's former graduate student, invented the first yellow LED and 10x brighter red and red-orange LEDs. Shuji Nakamura of Nichia Corporation of Japan demonstrated the first high brightness blue LED based on InGaN. The 2006 Millennium Technology Prize was awarded to Nakamura for his invention.
\end{abstract}

A Light emitting diode (LED) is essentially a pn junction diode. When carriers are injected across a forward-biased junction, it emits incoherent light. Most of the commercial LEDs are realized using a highly doped $\mathrm{n}$ and a $\mathrm{p}$ Junction.

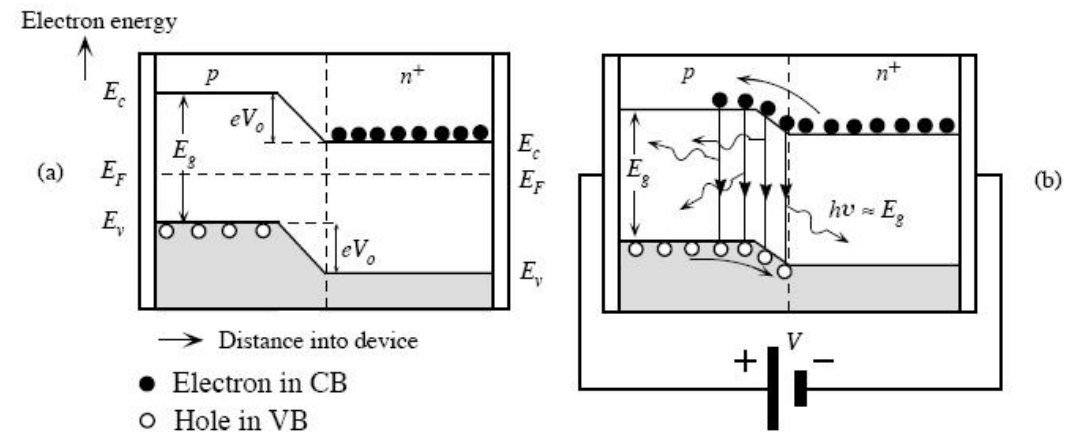

(a) The energy band diagram of a $\mathrm{pn}^{+}$(heavily $n$-type doped) junction without any bias. Built-in potential $V_{o}$ prevents electrons from diffusing from $n^{+}$to $p$ side. (b) The applied bias reduces $V_{o}$ and thereby allows electrons to diffuse or be injected into the $p$-side. Recombination around the junction and within the diffusion length of the electrons in the $p$-side leads to photon emission.

\section{Heading}

To understand the principle, let's consider an unbiased $\mathrm{pn}+$ junction. The depletion region extends mainly into the p-side. There is a potential barrier from Ec on the $\mathrm{n}$-side to the Ec on the p-side, called the builtin voltage, $\mathrm{V} 0$. This potential barrier prevents the excess free electrons on the $\mathrm{n}+$ side from diffusing into the $\mathrm{p}$ side. When a Voltage $\mathrm{V}$ is applied across the junction, the built-in potential is reduced from V0to V0 - V. This allows the electrons from the $\mathrm{n}+$ side to get injected into the $\mathrm{p}$-side. Since electrons are the minority carriers in the $\mathrm{p}$-side, this process is called minority carrier injection. But the hole injection from the $\mathrm{p}$ side to $\mathrm{n}+\mathrm{side}$ is very less and so the current is primarily due to the flow of electrons into the p-side. These electrons injected into the p-side recombine with the holes. This recombination results in spontaneous emission of photons (light). This effect is called injection electroluminescence. These photons should be allowed to escape from the device without being reabsorbed. The recombination can be classified into the following two kinds:

a. Direct recombination:

In direct band gap materials, the minimum energy of the conduction band lies directly above the maximum energy of the valence band in momentum space energy. In this material, free electrons at the bottom of the conduction band can recombine directly with free holes at the top of the valence band, as the momentum of the two particles is the same. This transition from conduction band to valence band involves photon 
emission (takes care of the principle of energy conservation). This is known as direct recombination. Direct recombination occurs spontaneously. GaAs is an example of a direct band-gap material.
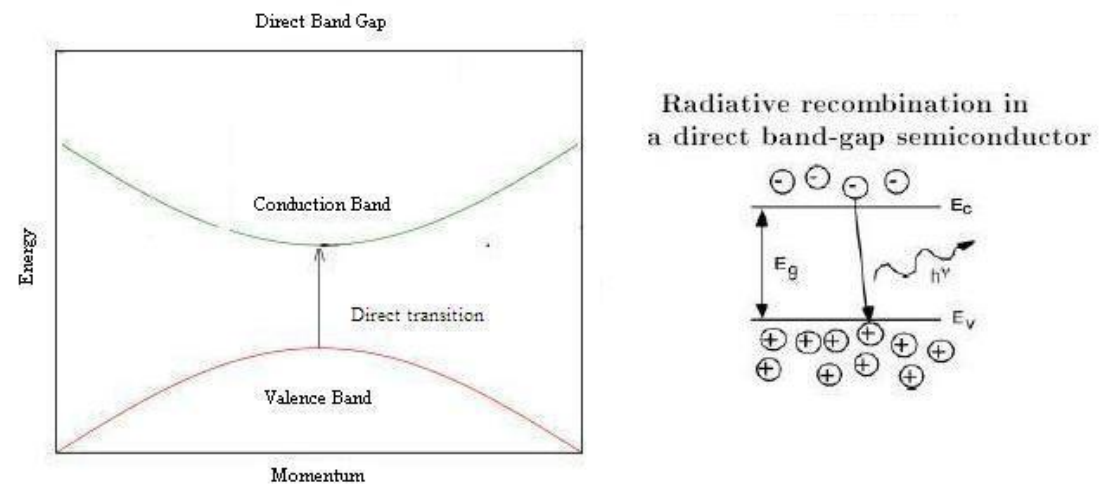

\section{b. In-Direct recombination}

In the indirect band gap materials, the minimum energy in the conduction band is shifted by a k-vector relative to the valence band. The k-vector difference represents a difference in momentum. Due to this difference in momentum, the probability of direct electron hole recombination is less. In these materials, additional dopants(impurities) are added which form very shallow donor states. These donor states capture the free electrons locally; provides the necessary momentum shift for recombination. These donor states serve as the recombination centers. This is called Indirect (non-radiative) Recombination.Figure 3 shows the E-k plot of an indirect band gap material and an example of how Nitrogen serves as a recombination center in GaAsP. In this case it creates a donor state when $\mathrm{SiC}$ is doped with $\mathrm{Al}$, it recombination takes place through an acceptor level. The indirect recombination should satisfy both conservation energy, and momentum. Thus besides a photon emission, phonon emission or absorption has to take place. $\mathrm{GaP}$ is an example of an indirect band-gap material.

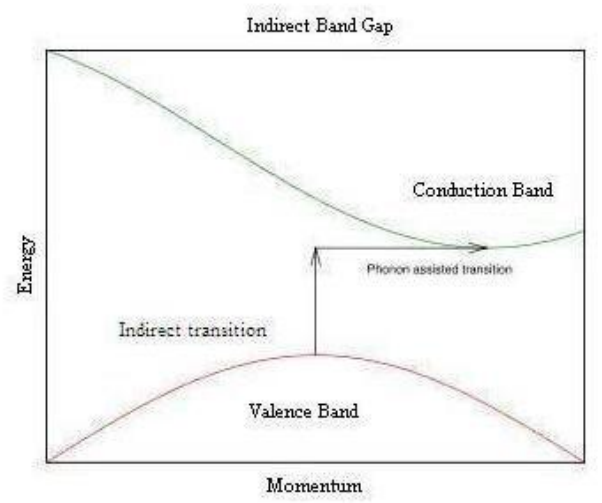

Addition of a nitrogen recombination center to indirectGaAsP

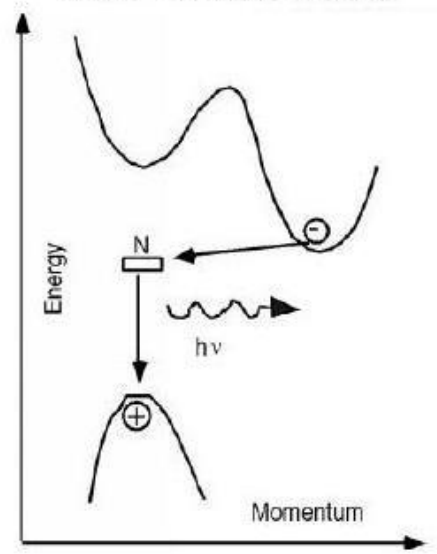

The wavelength of the light emitted, and hence the color, depends on the band gap energy of the materials forming the $\mathrm{p}$-n junction. The emitted photon energy is approximately equal to the band gap energy of the semiconductor. The following equation relates the wavelength and the energy band gap.

$$
\begin{aligned}
& \mathrm{h} v=\mathrm{Eg} \\
& \mathrm{h} \mathrm{c} / \lambda=\mathrm{Eg} \\
& \lambda=\mathrm{hc} / \mathrm{Eg} \\
& \text { Where } \mathrm{h} \text { is Plank's constant, } \mathrm{c} \text { is the speed of the light and Eg is the energy band gap }
\end{aligned}
$$

Thus, a semiconductor with a $2 \mathrm{eV}$ band-gap emits light at about $620 \mathrm{~nm}$, in the red. A $3 \mathrm{eV}$ band-gap material would emit at $414 \mathrm{~nm}$, in the violet. Appendix 4 shows a list of semiconductor materials and the corresponding colors.

\section{LED Materials:}

An important class of commercial LEDs that cover the visible spectrum are the III-V. Ternary alloys based on alloying GaAs and $\mathrm{GaP}$ which are denoted by GaAs1- 
yPy. InGaAlP is an example of a quarternary (four element) III-V alloy with a direct bandgap. The LEDs realized using two differently doped semiconductors that are the same materialis called a homo junction. When they are realized using different band gap materials they

are called a hetero structure device. A hetero structure LED is brighter than a homo Junction LED.

\section{LED Structure:}

The LED structure plays a crucial role in emitting light from the LED surface. The LEDsare structured to ensure most of the recombination takes place on the surface by the following two ways.

- By increasing the doping concentration of the substrate, so that additional free minority charge carriers electrons move to the top, recombine and emit light at the surface.

- By increasing the diffusion length $L=\sqrt{ } D \tau$, where $D$ is the diffusion coefficient and $\tau$ is the carrier life time. But when increased beyond a critical length there is a chance of re-absorption of the photons into the device.

The LED has to be structured so that the photons generated from the device are emitted without being reabsorbed. One solution is to make the $\mathrm{p}$ layer on the top thin, enough to create a depletion layer. Following picture shows the layered structure. There are different ways to structure the dome for efficient emitting.

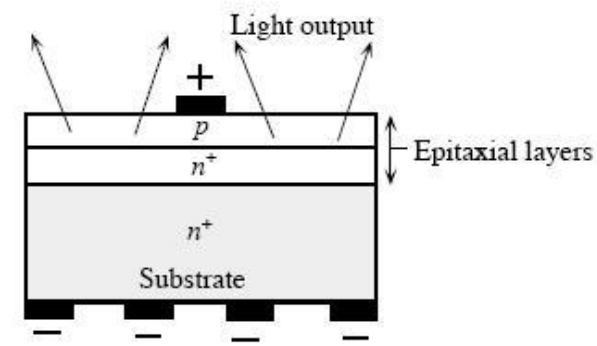

(a)

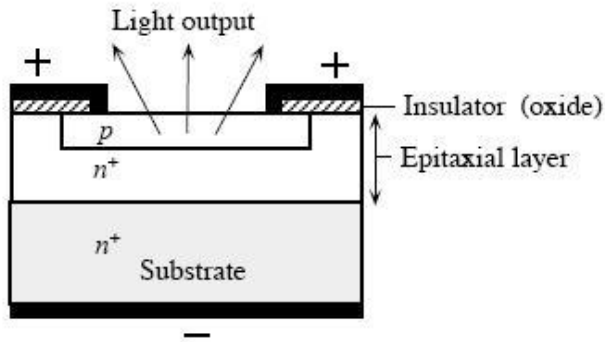

(b)

A schematic illustration of typical planar surface emitting LED devices. (a) $p$-layer grown epitaxially on an $n^{+}$substrate. (b) First $n^{+}$is epitaxially grown and then $p$ region is formed by dopant diffusion into the epitaxial layer.

LEDs are usually built on an n-type substrate, with an electrode attached to the p-type layer deposited on its surface. P-type substrates, while less common, occur as well. Many commercial LEDs, especially GaN/InGaN, also use sapphire substrate.

\section{LED efficiency:}

A very important metric of an LED is the external quantum efficiency $\eta$ ext. It quantifies the efficeincy of the conversion of electrical energy into emitted optical energy. It is defined as the light output divided by the electrical input power. It is also defined as the product of Internal radiative efficiency and Extraction efficiency.

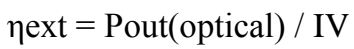

For indirect bandgap semiconductors next is generally less than $1 \%$, whereas for a direct band gap material it could be substantial.

ๆint $=$ rate of radiation recombination/ Total recombination

The internal efficiency is a function of the quality of the material and the structure and composition of the layer.

LED dome shapes: The LED domes are constructed such most of the light gets emitted efficiently. Following picture shows the two different kinds of domes. 
(a)

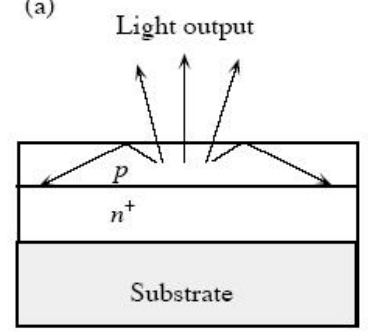

(b)

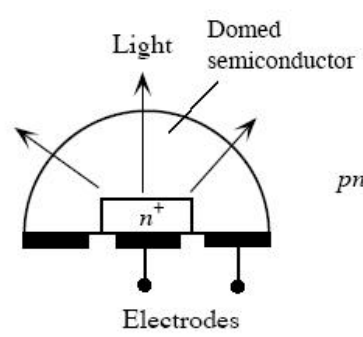

(c)

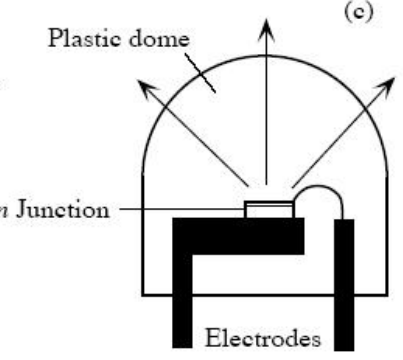

(a) Some light suffers total internal reflection and cannot escape. (b) Internal reflections can be reduced and hence more light can be collected by shaping the semiconductor into a dome so that the angles of incidence at the semiconductor-air surface are smaller than the critical angle. (b) An economic method of allowing more light to escape from the LED is to encapsulate it in a transparent plastic dome.

(a)

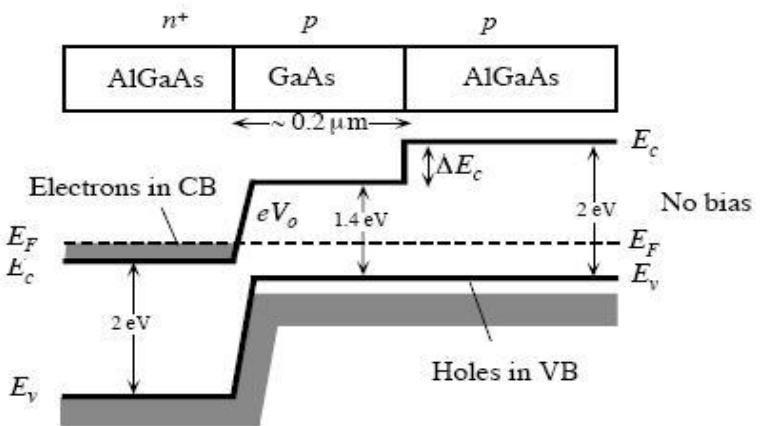

(c)

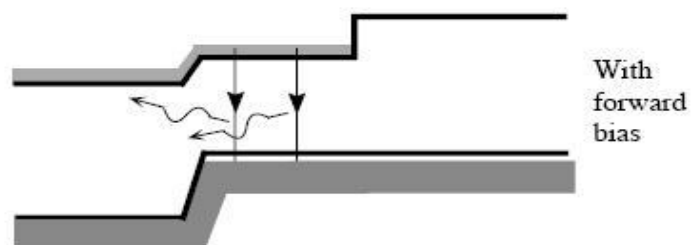

(a) A double

heterostructure diode has two junctions which are between two different bandgap semiconductors (GaAs and AlGaAs).

(b) A simplified energy band diagram with exaggerated features. $E_{F}$ must be uniform.

(c) Forward biased simplified energy band diagram.

(d) Forward biased LED. Schematic illustration of photons escaping

reabsorption in the $\mathrm{AlGaAs}$ layer and being emitted

(d)

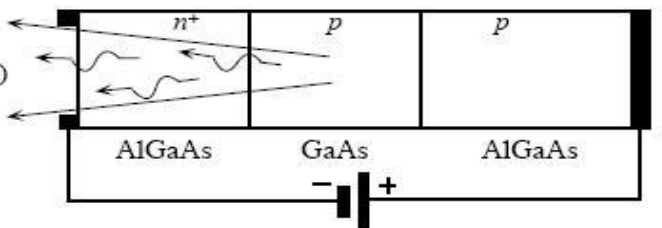

from the device.

III. Figures

Band gaps of some Common Semiconductors relative to the optical spectrum

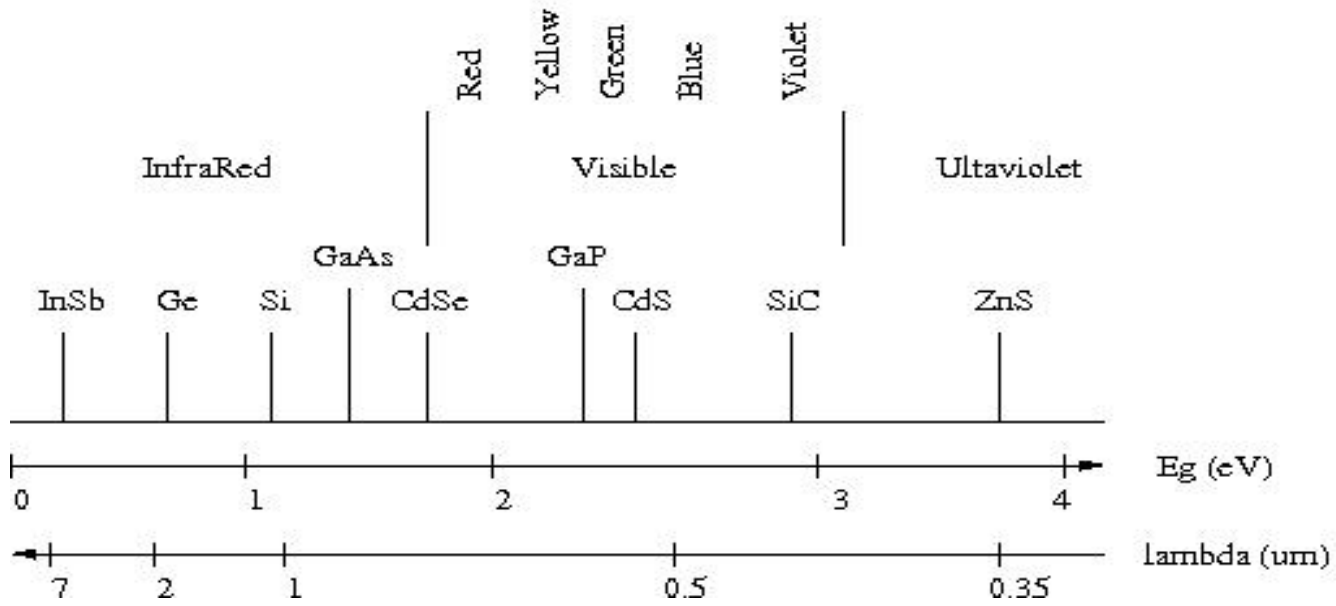




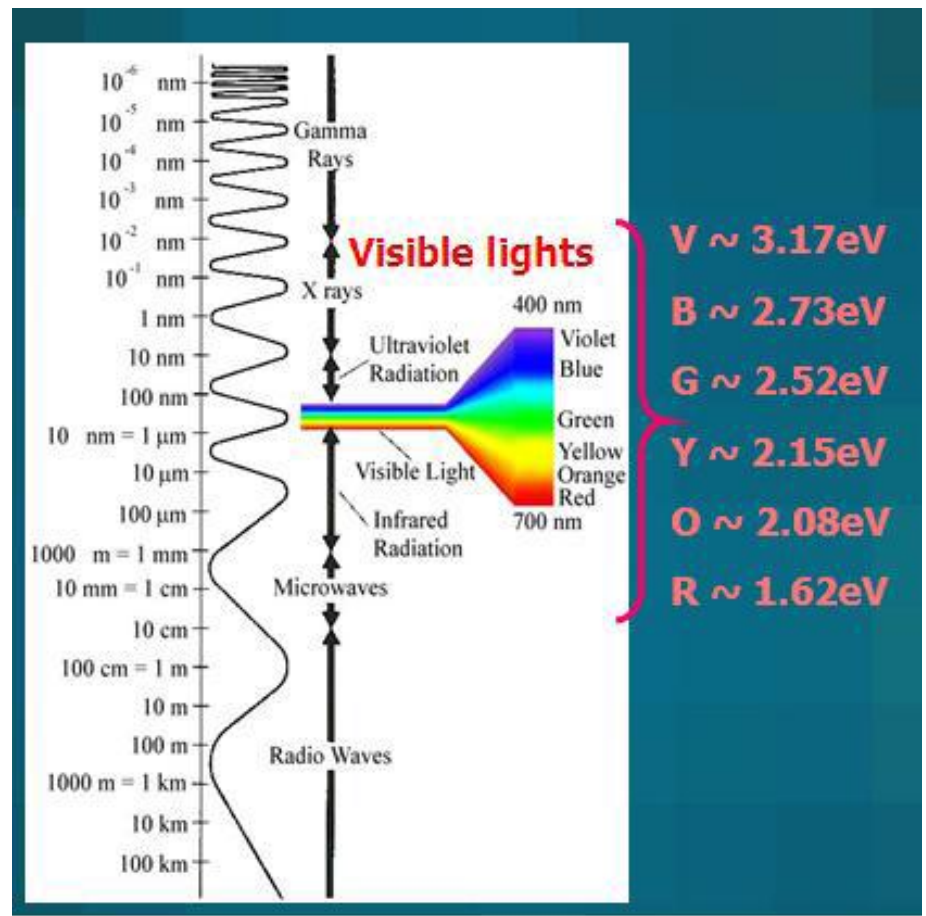

A heterostructure device. Following picture shows an example

(a)

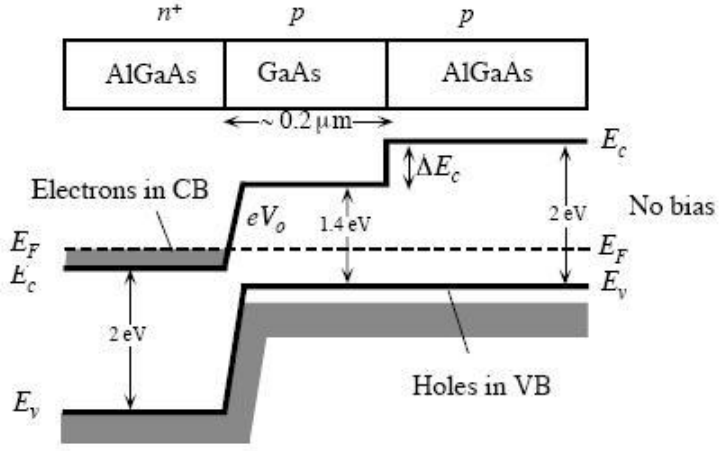

(c)

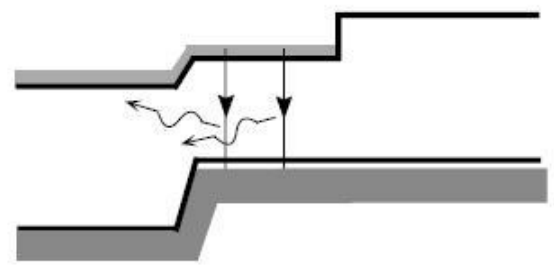

With

forward bias

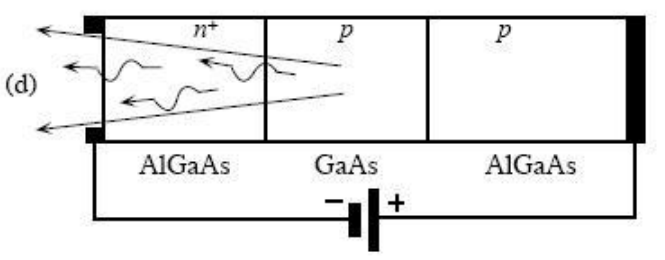

(a) A double

heterostructure diode has two junctions which are between two different bandgap semiconductors (GaAs and AlGaAs).

(b) A simplified energy band diagram with exaggerated features. $E_{F}$ must be uniform.

(c) Forward biased simplified energy band diagram.

(d) Forward biased LED. Schematic illustration of photons escaping reabsorption in the $\mathrm{AlGaAs}$ layer and being emitted from the device.

\section{Conclusions}

Applications: LED have a lot of applications. Following are few examples:

- Devices, medical applications, clothing, toys

- Remote Controls (TVs, VCRs)

- Lighting

- Indicators and signs

- Optoisolators and optocouplers 


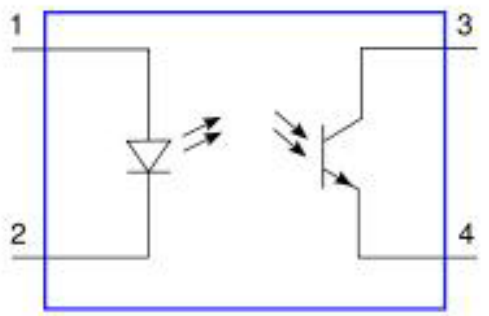

- Swimming pool lighting.

Advantages of using LEDs:

- LEDs produce more light per watt than incandescent bulbs; this is useful inbattery powered or energy-saving devices.

- LEDs can emit light of an intended color without the use of color filters that traditional lighting methods require. This is more efficient and can lower initialcosts.

- The solid package of the LED can be designed to focus its light. Incandescent andfluorescent sources often require an external reflector to collect light and direct itin a usable manner.

- When used in applications where dimming is required, LEDs do not change their color tint as the current passing through them is lowered, unlike incandescentlamps, which turn yellow.

- LEDs are ideal for use in applications that are subject to frequent on-off cycling,unlike fluorescent lamps that burn out more quickly when cycled frequently, or High Intensity Discharge (HID) lamps that require a long time before restarting.

- LEDs, being solid state components, are difficult to damage with external shock.Fluorescent and incandescent bulbs are easily broken if dropped on the ground.

- LEDs can have a relatively long useful life. A Philips LUXEON k2 LED has alife time of about 50,000 hours, whereas Fluorescent tubes typically are rated atabout 30,000 hours, and incandescent light bulbs at 1,000-2,000 hours.

- LEDs mostly fail by dimming over time, rather than the abrupt burn-out ofincandescent bulbs.

- LEDs light up very quickly. A typical red indicator LED will achieve fullbrightness in microseconds; Philips Lumileds technical datasheet DS23 for the

Luxeon Star states "less than 100ns." LEDs used in communications devices canhave even faster response times.

- LEDs can be very small and are easily populated onto printed circuit boards.

- LEDs do not contain mercury, unlike compact fluorescent lamps.

\section{Disadvantages:}

- LEDs are currently more expensive, price per lumen, on an initial capital costbasis, than more conventional lighting technologies. The additional expense

partially stems from the relatively low lumen output and the drive circuitry andpower supplies needed. However, when considering the total cost of ownership(including energy and maintenance costs), LEDs far surpass incandescent orhalogen sources and begin to threaten the future existence of compact fluorescentlamps.

- LED performance largely depends on the ambient temperature of the operatingenvironment. Over-driving the LED in high ambient temperatures may result inoverheating of the LED package, eventually leading to device failure. Adequateheat-sinking is required to maintain long life (See Appendix8 9).

- LEDs must be supplied with the correct current. This can involve series resistorsor current-regulated power supplies.

- LEDs do not approximate a "point source" of light, so they cannot be used inapplications needing a highly collimated beam. LEDs are not capable of providingdivergence below a few degrees. This is contrasted with commercial ruby laserswith divergences of 0.2 degrees or less. However this can be corrected by usinglenses and other optical devices.

- There is increasing concern that blue LEDs and white LEDs are now capable ofexceeding safe limits of the socalled blue-light hazard as defined in the eyesafety specifications for example ANSI/IESNA RP-27.1-05: Recommended Practice for Photo biological Safety for Lamp and Lamp Systems.

LEDs in the future: LEDs have come a long way and currently they are widely used inmany applications. In future, I believe research will continue for high intensity LEDs, even though heat dissipation is an issue(see appendix 8

Following is a list of semiconductor materials and the corresponding colors: 
- Aluminium gallium arsenide (AlGaAs) — red and infrared• Aluminium gallium phosphide ( $\mathrm{AlGaP})$ - green

- Aluminium gallium indium phosphide (AlGaInP) - high-brightness orange-red, orange, yellow, and green

- Gallium arsenide phosphide (GaAsP) — red, orange-red, orange, and yellow

- Gallium phosphide $(\mathrm{GaP})$ - red, yellow and green

- Gallium nitride (GaN) - green, pure green (or emerald green), and blue alsowhite (if it has an AlGaN Quantum Barrier)

- Indium gallium nitride ( $\mathrm{InGaN})-450 \mathrm{~nm}-470 \mathrm{~nm}$ - near ultraviolet, bluishgreenand blue

- Silicon carbide ( $\mathrm{SiC})$ as substrate - blue

- Silicon ( $\mathrm{Si}$ ) as substrate - blue (under development)

- Sapphire (Al2O3) as substrate - blue

- Zinc selenide (ZnSe) - blue

- Diamond (C) - ultraviolet

- Aluminium nitride (AlN), aluminium gallium nitride (AlGaN), aluminium gallium indium nitride (AlGaInN)

- near to far ultraviolet (down to $210 \mathrm{~nm}$ 